\title{
Numerical modeling of co-emissions for gas turbine combustors operating at part-load conditions
}

\section{Original article}

Article history:

Accepted: 8 May 2018

Published: 6 July 2018

Check for updates

*Correspondence:

NK: klarmannatd.mw.tum.de

\section{Peer review:}

Single blind

\section{Copyright:}

(c) 2018 Klarmann et al. () This is an open access article distributed under the Creative Commons Attribution License (CC-BY 4.0), which permits unrestricted use, distribution, and reproduction in any medium, provided the original work is properly cited and its authors credited.

\section{Keywords:}

computational fluid dynamics; gas turbine combustor; part-load operation;

CO-emissions; fuel staging

\section{Citation:}

Klarmann N., Zoller B. T., and Sattelmayer T. (2018). Numerical modeling of coemissions for gas turbine combustors operating at part-load conditions. Journal of the Global Power and Propulsion Society. 2: 376-387. https://doi.org/10.22261/JGPPS.C3N5OA

\author{
Noah Klarmann ${ }^{1 \star}$, Benjamin Timo Zoller ${ }^{2}$, Thomas Sattelmayer $^{1}$ \\ ${ }^{1}$ Lehrstuhl für Thermodynamik TU-München, 85748 Garching, Germany \\ ${ }^{2}$ GE Power, 5401 Baden, Switzerland
}

\begin{abstract}
A numerical model is presented for the precise prediction of carbon monoxide (CO) emissions in gas turbine combustors. All models are based on Computational Fluid Dynamics (CFD). This work starts with an introduction of fundamental mechanisms, which are responsible for $\mathrm{CO}$ emissions. As we will show, there is a need of CO-models as standard combustion models fail to predict $\mathrm{CO}$-emissions precisely. For the purpose of validation, experiments are conducted. High ratios of secondary air is bypassing the burner in order to induce interaction of the flame front with secondary air, as the flame brush gets diluted and decreases in reactivity. Note, this is an important mechanism for elevated $\mathrm{CO}$ emissions in multi-burner systems with high staging ratio. Five operating points with each having a different adiabatic flame temperature were measured. They include equilibrium (complete burnout) and superequilibrium $\mathrm{CO}$ (incomplete burnout). In summary, it is shown that the prediction of $\mathrm{CO}$ with the presented models lead to a significant improvement as it captures the transition from equilibrium to superequilibrium $\mathrm{CO}$. Furthermore, the strong underestimation of $\mathrm{CO}$ predicted by standard combustion models is shown.
\end{abstract}

\section{Introduction}

Extending the operational window is one of the main challenges in gas turbine development as operational flexibility is a key attribute to meet the requirements of tomorrow. Load decrease is limited by a sharp rise in CO-emissions caused by critically low flame temperature. The objective of this work is a CFD-based model, which is able to predict CO in combustion systems operating at part-load conditions. The model supports the development of combustion systems fulfilling future emissions legislations.

Standard combustion models typically face issues predicting $\mathrm{CO}$-emissions in gas turbine combustors. For example, the popular Flamelet Generated Manifold (FGM) model is based on the assumption, that a turbulent flame brush can be described by a set of laminar flamelets. A flamelet is a thin reaction zone dividing unburnt and burnt material. In flamelets, the late CO-oxidation is strongly increased due to the availability of a stable pool of radicals. In contradiction to the flamelet assumption, part-load combustion in gas turbines shows superequilibrium $\mathrm{CO}$ in the exhaust gas far behind the heat release zone. It is obvious that the source terms responsible for the burn out of $\mathrm{CO}$ cannot be described by flamelets. 
Nevertheless, the prediction of CO using FGM can be found in Goldin et al. (2012). Here, the authors used a combination of FGM and a turbulent flame speed model to close the reaction progress source term. The author concludes that FGM drastically overestimates the source terms of CO-oxidation.

Another approach can be found in Wegner et al. (2011). Here, CO is described by its own transport equation. Within the turbulent flame brush, $\mathrm{CO}$ is set to the maximum value of $\mathrm{CO}$ at a predefined reaction progress state. The peak value of $\mathrm{CO}$ is determined by flamelet calculations. The source term describing the CO-burnout is closed by detailed chemistry.

On the basis of literature review, industrial requirements and fundamental studies, we identified the following key elements for our approach:

- Turbulence: Due to the technical relevance of this work, we focus on efficient models in order to ensure applicability for industrial applications. Hence, we decided to build the model on the basis of Reynolds-averaged Navier-Stokes (RANS) equations. Note that the presented modeling strategy is not limited to RANS.

- Combustion: Simulation of combustors operating at part-load conditions requires advanced strategies in combustion modeling. As the prediction of $\mathrm{CO}$ strongly depends on a precise heat release distribution, the FGM Extension model is used (published by the authors in Klarmann et al. (2016)). The advantage of this model is that it is validated on flames which can be characterized by low reactivity. Here, it is inevitable to consider flame stretch and heat loss as it may substantially alter shape and position of the turbulent flame brush. Note that the implementation is able to consider partially premixed combustion. This is of great significance as $\mathrm{CO}$ is sensitive to dilution by secondary air.

- CO-Model: As already discussed, separating time scales of combustion and late burnout is necessary. As basic studies revealed, the impact of flame stretch and heat loss to CO cannot be neglected. Flame stretch substantially lowers the peak value of $\mathrm{CO}$ within the turbulent flame brush. Furthermore, heat loss cannot be neglected as it may substantially decreases the CO-oxidation which is calculated using the temperature dependent Arrhenius law.

\section{Numerical model for CO-emissions}

The Favre-averaged transport equation for a generic variable $\tilde{\phi}$ reads:

$$
\frac{\partial(\bar{\rho} \tilde{\Phi})}{\partial t}+\frac{\partial}{\partial x_{i}}\left(\bar{\rho} \tilde{u}_{i} \tilde{\Phi}-\bar{\rho} \frac{\mu_{t}}{S c_{t}} \frac{\partial \tilde{\Phi}}{\partial x_{i}}\right)=\overline{\dot{\omega}_{\Phi}}
$$

The terms on the left-hand side (transient term and transport terms) are closed in the context of RANS when considering the generally employed gradient diffusion approach. The Reynolds-Averaged source term on the right-hand side remains unclosed.

This work is using two different approaches to (1) model the combustion by modifying the reaction progress source term closure of FGM $(\tilde{\phi}=\tilde{c})$ and (2) modeling $\mathrm{CO}\left(\tilde{\phi}=\tilde{\mathrm{Y}}_{\mathrm{CO}}\right)$. Both models are introduced in the following two sections.

\section{Combustion model: FGM extension}

This section is a short summary of the model published by the authors in Klarmann et al. (2016). It is based on the FGM model initially published by van Oijen and de Goey (2002). In the context of FGM, the transport equation for the reaction progress $\tilde{c}$ is closed by the PDF-integrated source term obtained from one-dimensional flamelet calculations:

$$
\overline{\dot{\omega}_{c}^{0}}=\iint \dot{\omega}_{c}^{0}(c, f) P(c) P(f) \mathrm{d} c \mathrm{~d} f
$$

Here, turbulence is considered by integrating the product of the reaction progress source term and the probability density function $P$. For the evaluation of $P$ presumed beta-functions are used. This requires additional transport equations for the variance of every control variable $(c$ and $f$ ). The model extension modifies the reaction progress source term closure of FGM by a correction factor $\Gamma$. The PDF-integrated 
source term then reads:

$$
\overline{\dot{\omega}_{c}^{*}}=\Gamma(\kappa, \psi, f) \iint \dot{\omega}_{c}^{0}(c, f) P(c) P(f) \mathrm{d} c \mathrm{~d} f
$$

Here, $\Gamma$ is formulated on the basis of flame speeds:

$$
\Gamma(\kappa, \psi, f)=\left(\frac{s_{c}^{*}(\kappa, \psi, f)}{s_{c}^{0}(f)}\right)^{m}
$$

The underlying modeling idea is to divide the unstretched, adiabatic source term $\overline{\dot{\omega}_{c}^{0}}$ by its corresponding flame speed $s_{c}^{0}$ and to multiply the flame speed at the evaluated flame stretch and heat loss $s_{c}^{*}$. It can be shown that this is valid if the following relation was used to determine $m$ :

$$
s_{c}^{*}(\kappa, \psi, f)^{m} \sim{\overline{\omega_{c}^{*}}}_{c}(\kappa, \psi, f, c)
$$

More details on the determination of $m$ can be found in Klarmann et al. (2016).

\section{CO-model}

Mass fraction of CO is represented by its own transport equation. Wegner et al. (2011) initially proposed the idea to separate the time scales of CO-burnout from the combustion process. This idea is adopted in this work as we divide the domain in three regions: (1) preflame (inert), (2) inflame and (3) postflame as shown in the upper half of Figure 1. The employed assumption is that $\mathrm{CO}$ decouples from the combustion model under specific conditions. This leads to a burnout region behind the turbulent flame brush. Hence, the closure consists of two parts in which $\mathrm{CO}$ is treated differently (the preflame region does not require any modeling). Classification in inflame or postflame region is performed by estimating the limiting factor, which can be either turbulence (inflame) or the chemical finite rate of the oxidation of CO (postflame). This is based on the assumption that the inflame situation is dominated by the time scales of turbulent mixing (as chemistry is fast) and the burnout region is dominated by chemical time scales. The lower part of Figure 1 shows the CO-modeling strategy. CO is described to a certain point using FGM. After a decoupling event, $\mathrm{CO}$ is described by a burnout model providing substantially lower source terms due to the absence of radicals. All submodels are described in the following sections.

\section{Inflame model for $\mathrm{CO}$}

Within the turbulent flame brush, CO-chemistry is fast and interaction with turbulence cannot be neglected. Hence, we tabulate CO on the basis of PDF-integrated profiles of flamelets. CO cannot be accurately represented by flamelets without considering flame stretch and heat loss. Stretch alters diffusion of heat and species which strongly impacts CO. For example, a constant pressure reactor, fully neglecting diffusion, shows significantly

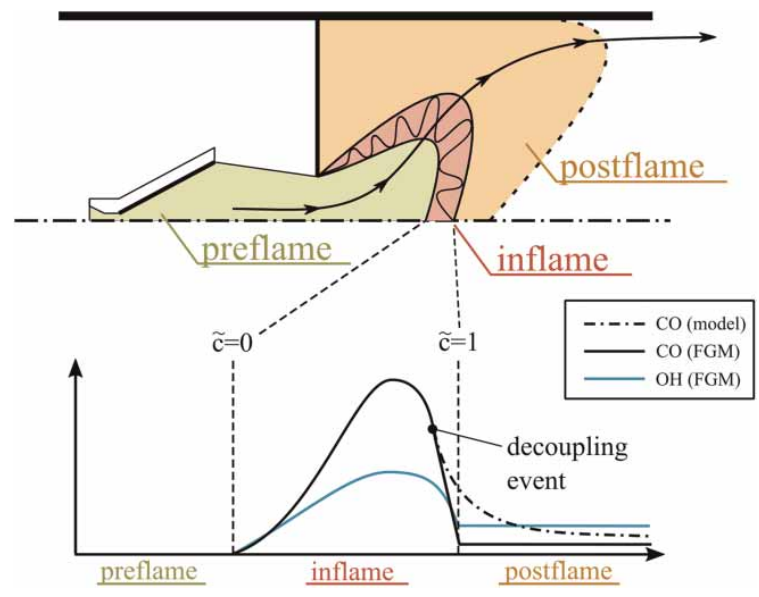

Figure 1. Decoupling mechanism of CO. 
higher CO compared to corresponding freely propagating flamelets. Adding the influence of flame stretch using premixed counter flow flamelets steepens the gradients of species and temperature and increases the effect of diffusion. The impact of stretch to CO is illustrated in Figure 2. Note that the impact of heat loss on CO profiles is lower than the impact of flame stretch. Adding stretch and heat loss as additional dimensions to the tabulation process would significantly increase the numerical effort. Therefore, an alternative is presented to model the influence of flame stretch and heat loss. The approach is similar to the correction factor used in the FGM Extension. The tabulation of $\mathrm{CO}$ on the basis of unstretched adiabatic flamelets reads:

$$
\overline{Y_{\mathrm{CO}}^{0}}=\iint Y_{\mathrm{CO}}^{0}(c, f) P(c) P(f) \mathrm{d} c \mathrm{~d} f
$$

As the proportionality between flame speed and heat loss differs from the proportionality between flame speed and stretch, we introduce two correction factors to consider both effects independently:

$$
\begin{gathered}
\overline{Y_{\mathrm{CO}}^{*}}=\Gamma_{\psi} \Gamma_{\kappa} \iint Y_{\mathrm{CO}}^{0}(c, f) P(c) P(f) \mathrm{d} c \mathrm{~d} f \\
\Gamma_{\psi}\left(\kappa^{0}, \psi, f\right)=\left(\frac{s_{c}^{*}\left(\kappa^{0}, \psi, f\right)}{s_{c}^{0}(f)}\right)^{n} \\
\Gamma_{\kappa}\left(\kappa, \psi^{0}, f\right)=\left(\frac{s_{c}^{*}\left(\kappa, \psi^{0}, f\right)}{s_{c}^{0}(f)}\right)^{o}
\end{gathered}
$$

This is analytically correct if the following relations are valid:

$$
\begin{aligned}
s_{c}^{*}\left(\kappa^{0}, \psi, f\right)^{n} & \sim \overline{Y_{\mathrm{CO}}^{*}}\left(\kappa^{0}, \psi, f, c\right) \\
s_{c}^{*}\left(\kappa, \psi^{0}, f\right)^{o} & \sim \overline{Y_{\mathrm{CO}}^{*}}\left(\kappa, \psi^{0}, f, c\right)
\end{aligned}
$$

Direct modeling of these relations is complex. Therefore, we introduce the assumption that the analytical relation is similar to the proportionality between flame speed and the peak value of CO before PDF-integration:

$$
\begin{aligned}
& s_{c}^{*}\left(\kappa^{0}, \psi, f\right)^{n} \sim Y_{\mathrm{CO}, \text { max }}^{*}\left(\kappa^{0}, \psi, f, c\right) \\
& s_{c}^{*}\left(\kappa, \psi^{0}, f\right)^{o} \sim Y_{\mathrm{CO}, \text { max }}^{*}\left(\kappa, \psi^{0}, f, c\right)
\end{aligned}
$$

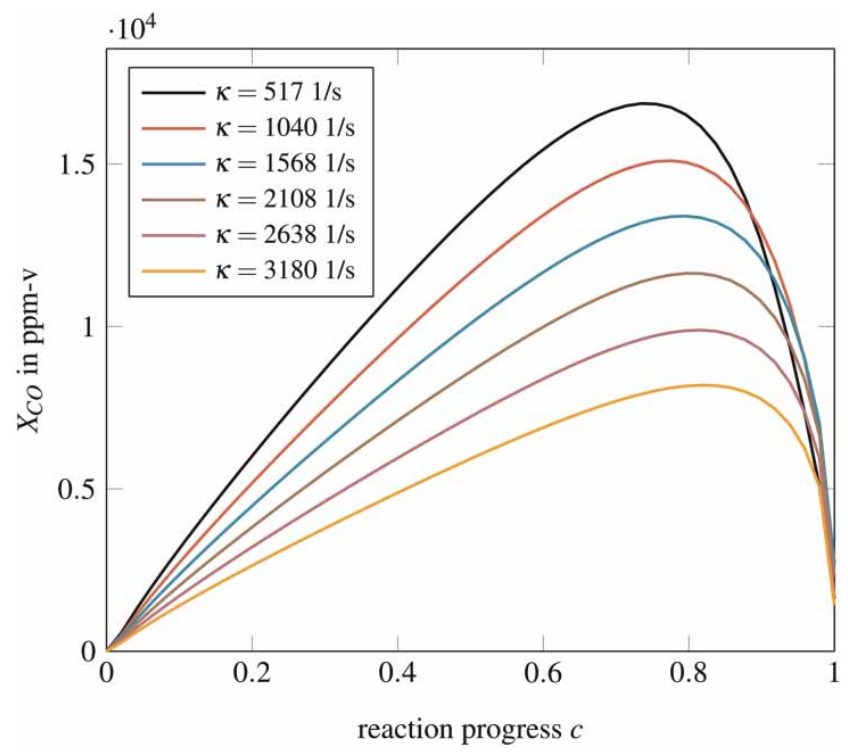

Figure 2. Influence of flame stretch on CO. 
Finally, the proportionality exponents read:

$$
\begin{aligned}
& n \sim \frac{\log \left(Y_{\mathrm{CO}, \max }^{*}\left(\kappa^{0}, \psi, f, c\right)\right)}{\log \left(s_{c}^{*}\left(\kappa^{0}, \psi, f\right)\right)} \\
& o \sim \frac{\log \left(Y_{\mathrm{CO}, \max }\left(\kappa, \psi^{0}, f, c\right)\right)}{\log \left(s_{c}^{*}\left(\kappa, \psi^{0}, f\right)\right)}
\end{aligned}
$$

The proportionality exponents are the gradient of a functional correlation between $\log \left(Y_{\mathrm{CO}, \max }\right)$ and $\log \left(s_{c}\right)$. Figure 3 shows the linear character of both relations. Consequently, both exponents are constant for varying heat loss and flame stretch and can be determined using a curve fit optimization assuming a linear equation. Note that the gradient for stretch correction is much steeper than for heat loss correction. This indicates that CO is impacted more by flame speed reduction based on flame stretch than on flame speed reduction based on heat loss.

\section{Postflame model for CO}

$\mathrm{CO}$-oxidation in the late burnout (i.e. in the postflame zone) can be described using a single reaction equation:

$$
\mathrm{CO}+\mathrm{OH} \rightarrow \mathrm{CO}_{2}+\mathrm{H}
$$

The proposed CO-model is based on the idea that behind the turbulent flame brush $\mathrm{H}$ and $\mathrm{OH}$ radicals are in equilibrium. This assumption is based on the fact that the chemical timescales of all radicals are orders of magnitude smaller than these of the CO-burnout. Hence, the postflame source term of $\mathrm{CO}$ is calculated using the equilibrium of $\mathrm{OH}$ :

$$
-\frac{\mathrm{d}[\mathrm{CO}]}{\mathrm{d} t}=k_{\mathrm{arr}, \mathrm{CO}}(T)[\mathrm{CO}][\mathrm{OH}]_{\mathrm{eq}}
$$

Figure 4 shows the experimental source term for two operating conditions of different adiabatic flame temperatures. Note that the source terms are based on the spatial gradient of $\mathrm{CO}$ which can be derived from measurements. The experimental setup can be find the following section. Furthermore, the corresponding source terms calculated by the introduced postflame model is plotted. Downstream of the $199 \mathrm{~mm}$ measurement position, the postflame model prediction and the experiments are in good agreement. Furthermore, Figure 4 shows the CO source terms of a freely propagating flamelet and a constant pressure reactor at the corresponding reaction progress (derived from experimentally measured $\mathrm{CO}_{2}$ and $\mathrm{CO}$ ). As already discussed, they clearly overestimate burnout rates of $\mathrm{CO}$.

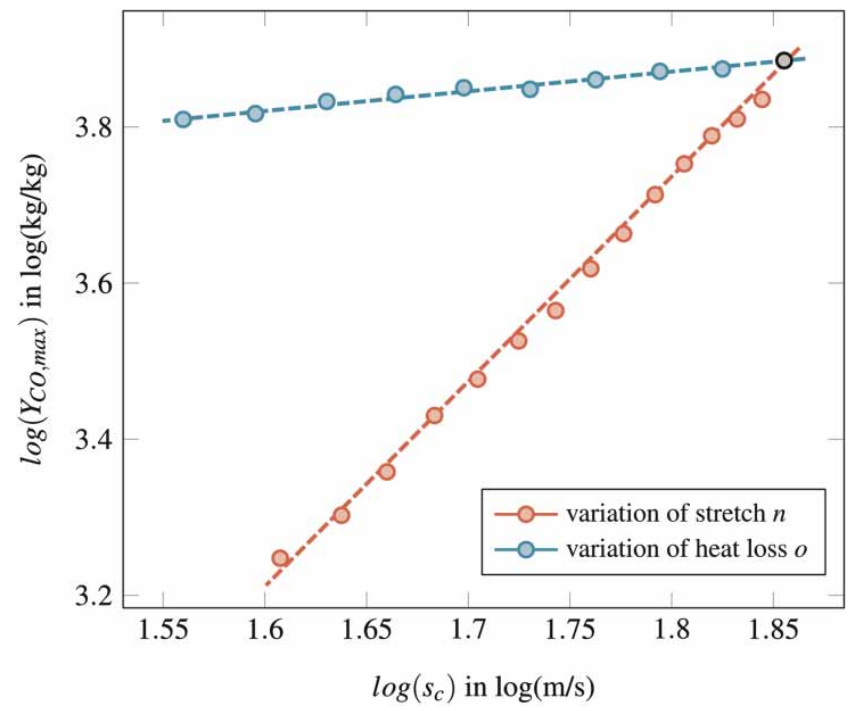

Figure 3. Functional correlation between logarithm of CO maximum and the logarithm of flame speed. 


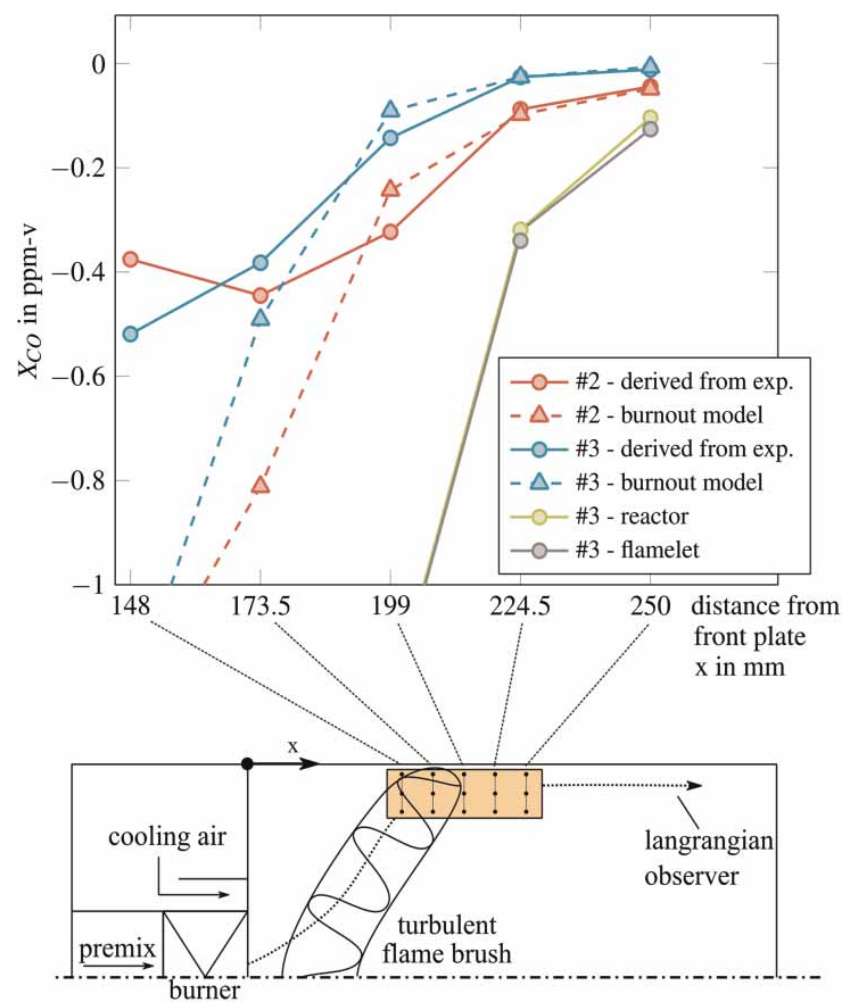

Figure 4. Comparison of experimentally derived and modeled source terms of CO.

\section{Modeling the transition}

The Transition model is based on an estimation of the turbulent Damköhler number for CO, which compares turbulent with chemical time scales:

$$
D a_{\mathrm{CO}}=\frac{\tau_{\text {turb }}}{\tau_{\mathrm{CO}}}
$$

We use the following expression which is the time needed to oxidize CO close to equilibrium:

$$
\tau_{\mathrm{CO}}=\frac{\ln \left(\frac{[\mathrm{CO}]_{\mathrm{eq}}}{[\mathrm{CO}]}\right)}{\left([\mathrm{CO}]-[\mathrm{OH}]_{\mathrm{eq}}\right) k_{\mathrm{arr}, \mathrm{CO}}}
$$

Multiple definitions for turbulent timescales exist. We decide to use a timescale characterizing eddies of the integral length scale (Poinsot and Veynante, 2005):

$$
\tau_{\text {turb }}=\frac{k}{\epsilon}
$$

This quantity is often used in terms of limiting combustion (e.g. in the Eddy-Break-up hypotheses of Spalding (1971)) as it characterizes the timescale of turbulent mixing.

The decoupling event takes place if $D a_{\mathrm{CO}}$ falls below a critical value:

$$
D a_{\mathrm{CO}}<D a_{\mathrm{CO}, \text { crit }}
$$

We experienced that this decoupling criterion is robust as there exists a transitional range where the burnout rate of $\mathrm{CO}$ for both inflame and postflame model are very close to each other. $D a_{\mathrm{CO}}$,rit should be chosen around unity as this marks the transition point when chemical timescales start exceeding turbulent timescales. 


\section{Experimental setup}

Experiments are conducted in an atmospheric single burner test rig with a thermal power of $50 \mathrm{~kW}$. Details of the burner can be found in Sangl (2011). The setup is depicted in Figure 5. An electrical preheater is providing air at a temperature of $300{ }^{\circ} \mathrm{C}$. Within the burner plenum, air is divided into primary air, which is swirled by the burner, and secondary air. The secondary air is bypassing the burner as it flows through a perforated plate into the combustion chamber. Fuel is injected at the burner slots.

Within the combustion chamber, swirling fuel-air mixture generates a vortex breakdown, where the flame stabilizes at the stagnation point. The outer part of the turbulent flame brush is diluted by secondary air, providing a region of strongly decreased reactivity, leading to elevated CO-emissions. Ceramic insulation prevents significant heat losses, which leads to similar conditions as present in gas turbine combustors. Furthermore, the chamber is cooled by impinging air. Local measurements are conducted using a water-cooled probe, which can be traversed in radial direction. Furthermore, the probe can be attached to multiple ports, which are located at several downstream positions. This enables the measurement of a two-dimensional grid. Note that due to the fact that the combustion chamber is cylindrical, we can assume rotational symmetry. This is advantageous as a two-dimensional grid measurement is sufficient to derive a volumetric distribution. We checked this assumption by comparing multiple $\mathrm{CO}$-profiles over radius of same axial positions. A gas analyzer is used to determine mole fractions of $\mathrm{CO}_{2}, \mathrm{O}_{2}, \mathrm{NO}_{\mathrm{x}}$, $\mathrm{CO}$ from the extracted gas samples. In order to evaluate heat loss precisely, temperature is monitored at the outer shell of the insulation using thermocouples. Measurements are conducted at five different adiabatic flame temperatures:

\#1-3: Superequilibrium CO.

\#4: Transition of incomplete burnout and equilibrium.

\#5: $\mathrm{CO}$ is in equilibrium.

Figure 6 depicts $\mathrm{CO}$ as a function of adiabatic flame temperature. Note, $\mathrm{CO}$ is measured at a characteristic residence time of about $20 \mathrm{~ms}$.

\section{Validation}

All introduced models are implemented in Fluent (Ansys, 2014) using a C-based interface. Tables are used to provide the required model input during runtime. All table entries are quantities derived from equilibrium calculations as well as premixed counter flow flamelets at predefined stretch rates, enthalpy defects, and mixture fractions. The preprocessing of the tabulated data is performed using a Python-based routine on the basis of the chemical kinetic software Cantera (Goodwin et al., 2015). Around 5,000 flamelet calculations have been used to generate the table. Table 2 lists some information of the CFD setup. Geometry is shown in Figure 7 and boundary conditions are listed in Table 1 . The domain is a quarter of the original geometry using periodical boundary

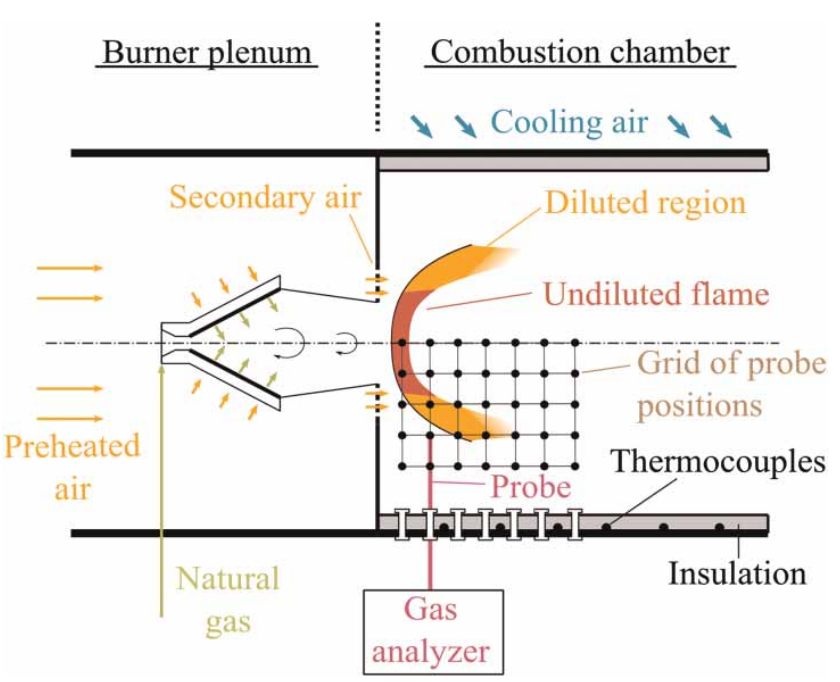

Figure 5. Scheme of the atmospheric single burner test rig. 


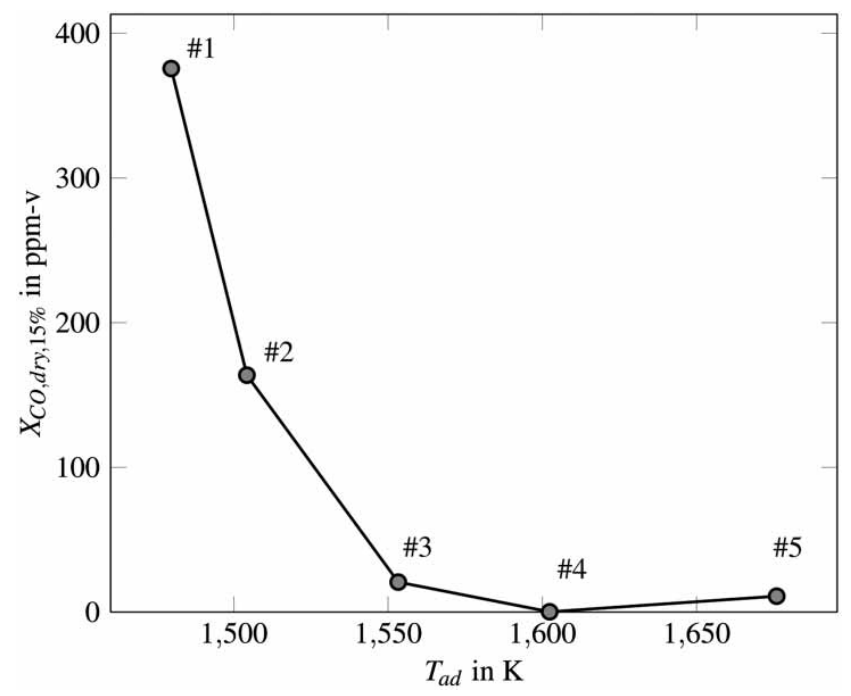

Figure 6. $\mathrm{CO}$ at a characteristic probe position at a residence time of about $20 \mathrm{~ms}$.

conditions. Note that the precise evaluation of wall heat loss is crucial to predict CO-burnout due to its temperature sensitivity. The non-adiabatic boundary condition is evaluated using temperature profiles measured at the outer shell of the insulation. We decided to use transient (URANS) simulations as we experienced unsteadiness using steady-state simulations. All results shown in the following are time averaged from the transient results.

Figure 8 illustrates experimental and numerical contour plots of $\mathrm{CO}$ of operating point \#4. The heat release distribution (indicated by $X_{\mathrm{CO}_{2} \text {,dry }}$ ) from the original implementation of FGM in Fluent (1) and the FGM Extension (2) show large differences in terms of position and shape of the turbulent flame brush. Comparing

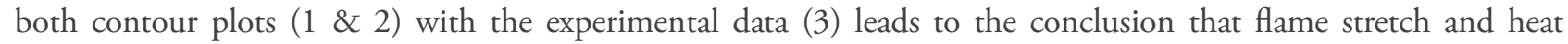
loss cannot be neglected. (4) shows the PDF-integrated value of $X_{\mathrm{CO} \text {,dry }}$ without any scaling by the introduced correction factors (Eq. 6). It clearly shows the overestimation when heat loss and stretch is not considered as the peak value in the turbulent flame brush is around three times higher than indicated by the experimental data (6). Contour plot (5) presents the solution using the introduced CO-model. Good agreement with the experimental data (6) in terms of maximum value of $\mathrm{CO}$ is evident. Furthermore, a burnout region develops behind the turbulent flame brush as it can be seen in the experimental data.

For the purpose of quantitative validation, it is reasonable to compare surface-averaged quantities as a probe position with high radius represents more surface than a position with low radius. Hence, we accumulate the product of $\mathrm{CO}$ and its corresponding ring face and divide the product by the total surface:

$$
\left\langle X_{\mathrm{CO}, \mathrm{dry}}\right\rangle_{\mathrm{area}}=\frac{\sum_{r} A_{r}\left\langle X_{\mathrm{CO}, \mathrm{dry}}\right\rangle_{r}}{A_{\text {sum }}}
$$

Figure 9 compares averaged $\mathrm{CO}$ values of experiments and simulations at three different axial position: $x=\{199 \mathrm{~mm}, 224.5 \mathrm{~mm}, 250 \mathrm{~mm}\}$. The corresponding residence times are estimated from CFD streamline

Table 1. Test rig operating conditions.

\begin{tabular}{|l|l|}
\hline$\dot{m}_{\text {air,tot }}$ & $32 \mathrm{~g} / \mathrm{s}$ \\
\hline$\dot{m}_{\text {air,sec }} / \dot{m}_{\text {air,pri }}$ & $\approx 30 \%$ \\
\hline$T_{\text {preh. }}$ & $573.15 \mathrm{~K}$ \\
\hline$p$ & $1 \mathrm{~atm}$ \\
\hline
\end{tabular}


Table 2. Numerical setup.

\begin{tabular}{|c|c|}
\hline \multicolumn{2}{|l|}{ Table Generation } \\
\hline Chemical mechanism & GRI 30 (Smith et al. 2014) \\
\hline Points of mixture fractions & 50 \\
\hline Points of enthalpy defects & 20 \\
\hline Points of strain rates & $<15$ \\
\hline Prop. exp. reaction progress $m$ & 1.5 \\
\hline Prop. exp. CO stretch $n$ & 2.0 \\
\hline Prop. exp. CO heat loss o & 0.37 \\
\hline \multicolumn{2}{|l|}{ CFD } \\
\hline Software & Fluent v15.0 (ANSYS Inc. 2014) \\
\hline Mesh (chamber only) & polyhedral, $2.4 \mathrm{e} 5$ cells \\
\hline$S c_{t}$ & 0.7 \\
\hline$D a_{\mathrm{CO}, \text { crit }}$ & 1.0 \\
\hline Periodicity & Quarter \\
\hline Turbulence closure & k-є realiz. (URANS) \\
\hline Pressure-velocity coupling & simple \\
\hline Upwind order & second order \\
\hline
\end{tabular}

analysis: $\tau_{\text {res }} \approx\{18 \mathrm{~ms}, 22 \mathrm{~ms}, 26 \mathrm{~ms}\}$. It can be seen that the original implementation of FGM strongly underestimates $\mathrm{CO}$. With decreasing adiabatic flame temperature, $\mathrm{CO}$ increases slightly as the heat release distribution is shifted more downstream in the vicinity of the wall. Using the FGM Extension and the CO-model leads to good agreement with the experimental data. The numerically predicted $\mathrm{CO}$ fits experiments best for the shortest residence time of $18 \mathrm{~ms}$. For higher residence times, the discrepancy between measurements and modeled CO increases, especially for the leanest operating point. Here, unburnt hydrocarbons may play an important role. Note that they cannot be accurately captured by our modeling approach as the assumption of complete heat release within the turbulent flame brush is used.

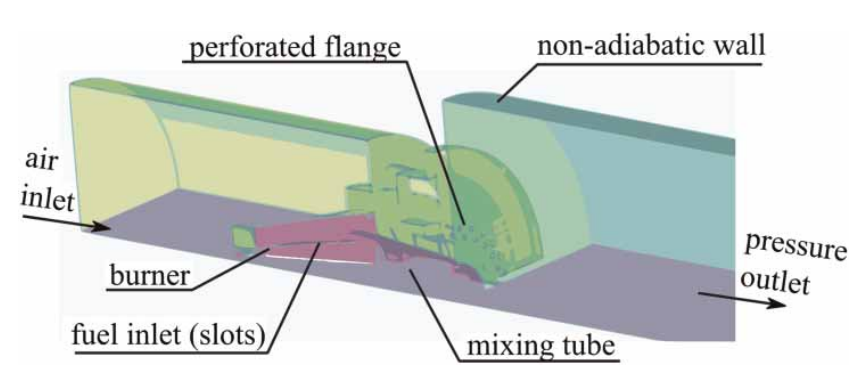

Figure 7. Geometry and boundary conditions. 

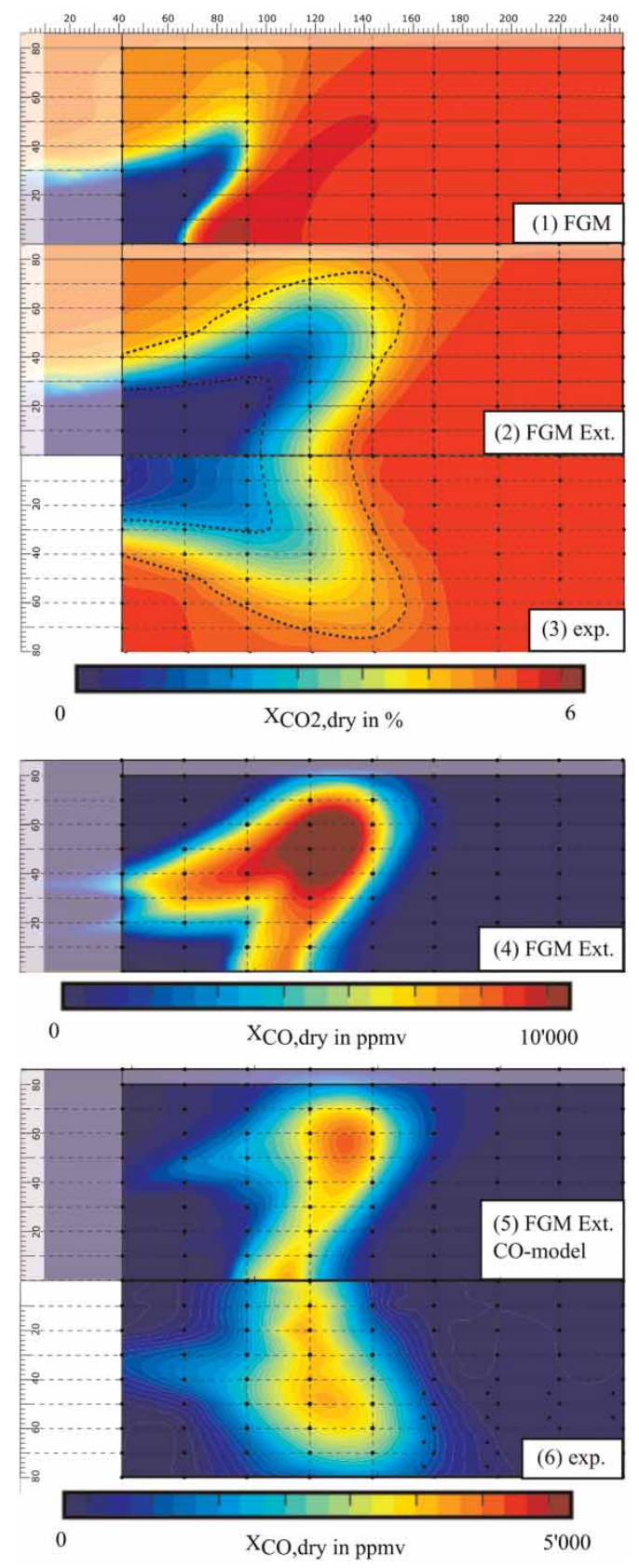

Figure 8. Numerical and experimental $\mathrm{CO}_{2}(1-3)$ and CO (5-6) of operation point \#4.

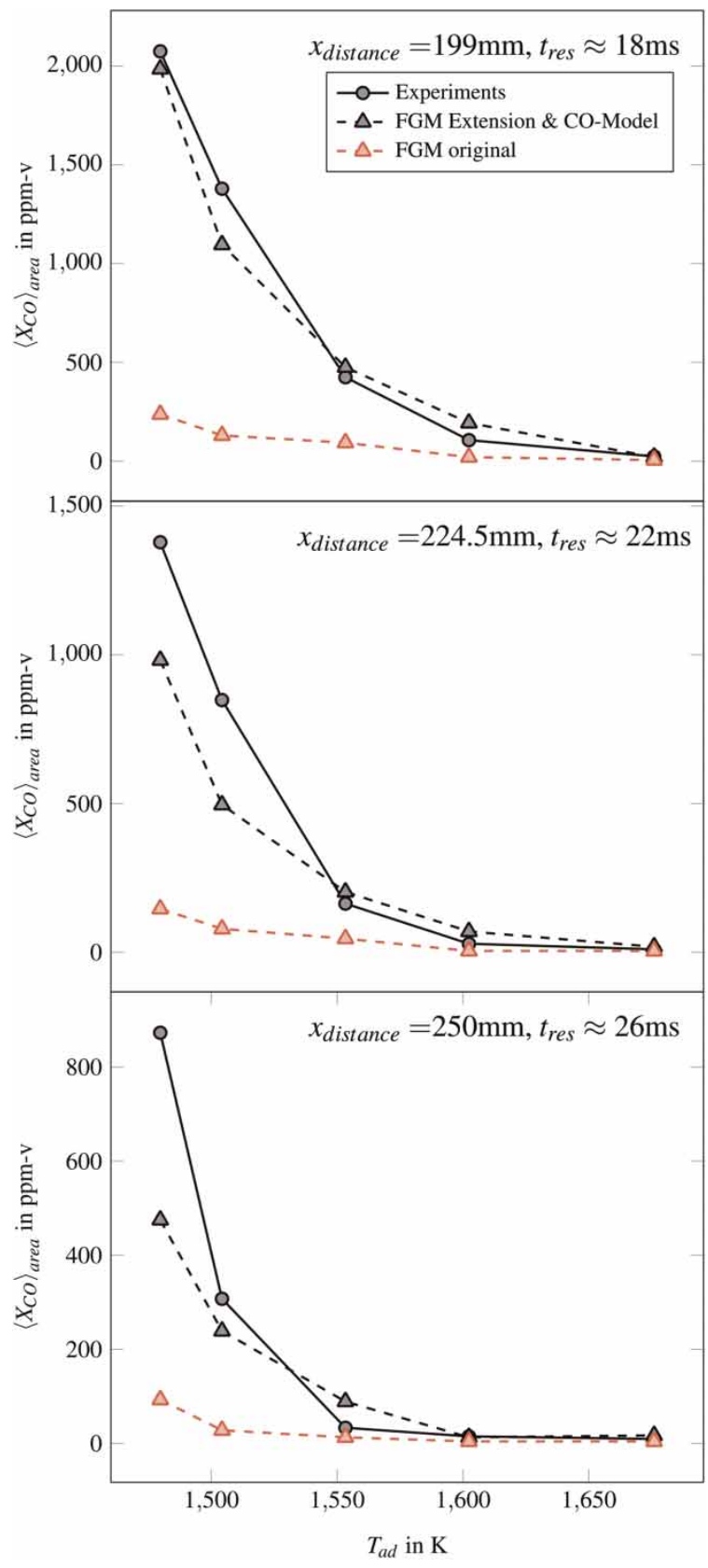

Figure 9. Comparison of experimental and numerical $\mathrm{CO}$ versus adiabatic flame temperature.

\section{Summary, conclusions, and outlook}

The paper presents an approach to predict CO-emissions numerically. The necessity of modeling CO on the basis of combustion models is discussed. Previous approaches and fundamental studies revealed that timescale separation between fast combustion and slow CO burnout is necessary. Hence, the proposed model is divided in an inflame and a postflame part. As we showed, inflame-CO cannot be described by tabulating freely propagating flamelets as flame stretch plays a major role. A model for the stretch and heat loss dependent correction of $\mathrm{CO}$ is presented. Furthermore, the source terms describing the late $\mathrm{CO}$ burnout behind the heat release zone is modeled by a single elementary reaction equation. Here, we introduced the model assumption that $\mathrm{OH}$ is in equilibrium after it decouples from the turbulent flame brush. This assumption was verified by comparing source terms derived from experiments with source terms calculated by the proposed postflame model.

All models are implemented in a commercial CFD-software and validated against experimental data. The numerically predicted $\mathrm{CO}$ agrees well with experimental data for different axial probe positions. Furthermore, we 
showed the strong underestimation of CO if FGM is used without any further modeling. This underlines the necessity of dedicated CO models to predict elevated CO-emissions.

Validation of high-pressure multi-burner configurations is planned in the future: In part-load operation, only a part of the burners are supplied with fuel. This leads to the situation that hot burners interact with cold air from the inactivated neighbouring burners.

\section{Nomenclature}

\section{Sub- and superscripts}

$\begin{array}{ll}(\ldots) & \text { Favre averaging } \\ (\ldots) & \text { Reynolds averaging } \\ (\ldots)^{0} & \text { Adiabatic and unstretched } \\ (\ldots) * & \text { Non-adiabatic and stretched } \\ (\ldots)^{\prime} & \text { Root mean square } \\ \langle\ldots\rangle_{\text {surf }} & \text { Surface average }\end{array}$

\section{Latin}

A Area, $\mathrm{m}^{2}$

c Reaction progress, -

$D a_{t}$ Turbulent Dammköhler number, -

$f$ Mixture fraction, -

$k_{\text {arr }}$ Reaction rate constant

$k$ Turbulent kinetic energy, $\mathrm{m}^{2} / \mathrm{s}^{2}$

$\dot{m}_{\text {air }}$ Mass flow of air, $\mathrm{kg} / \mathrm{s}$

$m$ Proportionality exponent for progress source term correction, -

$n$ Proportionality exponent for stretch correction, -

$o$ Proportionality exponent for non-adiabatic correction, -

$P \quad$ Probability density function, -

$p \quad$ Pressure, $\mathrm{Pa}$

Sc Turbulent Schmidt Number, -

$s_{c} \quad$ Fuel consumption speed, $\mathrm{m} / \mathrm{s}$

$t$ Time, $s$

$T$ Temperature, $\mathrm{K}$

$u$ Velocity, $\mathrm{m} / \mathrm{s}$

$x \quad$ Spatial coordinate, $\mathrm{m}$

$X_{i} \quad$ Mole fraction of species $i$, -

$Y_{i} \quad$ Mass fraction of species $i,-$

\section{Greek}

$\Gamma$ Correction factor

$\epsilon$ Turbulence dissipation rate, $\mathrm{m}^{2} / \mathrm{s}^{3}$

$\kappa$ Stretch, 1/s

$\mu_{t}$ Turbulent viscosity, $\mathrm{m} / \mathrm{s}^{2}$

$\rho$ Density, $\mathrm{kg} / \mathrm{m}^{3}$

$\tau$ Timescale, $s$

$\phi$ Generic variable

$\Psi$ Heat loss, $\mathrm{kJ} / \mathrm{kg}$

$\dot{\omega}$ Source term, $\mathrm{kg} / \mathrm{m}^{3} \mathrm{~s}$

\section{Funding sources}

The investigations were conducted as part of the joint research program COORETEC-turbo (AG Turbo 2020) in the frame of AG Turbo. The work was supported by the Bundesministerium für Wirtschaft und Technologie (BMWi) as per resolution of the German Federal Parliament under grant number 03ET7071H. The authors 
gratefully acknowledge AG Turbo and GE Power for their support and permission to publish this paper. The views and opinions expressed in this paper are those of the author only and do not necessarily reflect the views of or should they be attributed to General Electric Company.

\section{Competing interests}

All authors declare that they have no conflict of interest.

\section{References}

ANSYS Inc. (2014). ANSYS Fluent Academic Research, Release 15.0. ANSYS, Inc, Canonsburg, Pennsylvania, USA.

Goldin G., Ren Z., Forkel H., Lu L., Tangirala V., and Karim H. (2012). Modeling CO With Flamelet-Generated Manifolds: Part 2 Application. In: Proceedings of ASME Turbo Expo 2012, June 11-15, 2012, Copenhagen, Denmark.

Goodwin D., Malaya N., Moffat H., and Speth R. (2015) Cantera: An Object-Oriented Software Toolkit for Chemical Kinetics, Thermodynamics, and Transport Processes. Available at: code.google.com/p/cantera.

Klarmann N., Sattelmayer T., Geng W., and Magni F. (2016). Flamelet Generated Manifolds for Partially-Premixed, Highly-Stretched and Non-Adiabatic Combustion in Gas Turbines. In AIAA Science and Technology Forum and Exposition (SciTech 2016), January 4-8, San Diego, California, USA.

Poinsot T. and Veynante D. (2005). Theoretical and Numerical Combustion, 2nd ed. Philadelphia: Edwards.

Sangl J. (2011). Erhöhung der Brennstoffflexibilität von Vormischbrennern durch Beeinflussung der Wirbeldynamik. TU-München, Munich, Germany.

Smith G. P., Golden D. M., Frenklach M., Moriarty N. W., Eiteneer B., et al. (2014). GRI-Mech 3.0. Published at combustion. berkeley.edu/gri-mech/version30.

Spalding D. B. (1971). Mixing and chemical reaction in steady confined turbulent flames. Symposium (International) on Combustion. 13 (1): 649-657. https://doi.org/10.1016/S0082-0784(71)80067-X

van Oijen J. A., and de Goey L. P. H. (2002). Modelling of premixed counterflow flames using the flamelet-generated manifold method. Combustion Theory and Modelling. 6 (3): 463-478. https://doi.org/10.1088/1364-7830/6/3/305

Wegner B., Gruschka U., Krebs W., Egorov Y., Forkel H., et al. (2011). CFD prediction of partload CO emissions using a two-timescale combustion model. Journal of Engineering for Gas Turbines and Power. 133: 71502. https://doi.org/10.1115/GT2010-22241 